\title{
Prevenção de incapacidade física por hanseníase no Brasil: análise histórica
}

\author{
Prevention of physical disabilities due to leprosy in Brazil: \\ a historic analysis
}

Aleksandra Rosendo dos Santos (https://orcid.org/0000-0001-8104-0917) ${ }^{1}$

Eliane Ignotti (https://orcid.org/0000-0002-9743-1856) ${ }^{1}$

${ }^{1}$ Faculdade de Ciências da Saúde, Universidade do Estado de Mato Grosso. Av. Santos Dumont s/n, Cidade Universitária. 78.200000 Cáceres MT Brasil. alegramos@hotmail.com

\begin{abstract}
Guidelines for the prevention of physical disabilities due to leprosy have been gaining prominence in the control of the disease over and above multidrug therapy. The scope of this study is to describe the chronological changes in the technical guidelines for the prevention of physical disabilities due to leprosy in Brazil. It is a historical account based on official documents of the Brazilian health agencies. All the decrees, ordinances and manuals that mentioned assessment and prevention of disabilities published between 1962 and 2016 were included. The documents established the clinical, technical, welfare, emotional and social criteria that governed preventive actions in health institutions. The publications were coordinated by the Ministry of Health and adapted over the years from a conceptual, political, strategic and welfare standpoint. The guidelines mainly encompassed the activities of neurological assessment and the degree of physical incapacity, techniques of prevention of incapacities and self-care. Physical disabilities reflect the quality of access to diagnosis, monitoring of cases during treatment and post-discharge due to cure. The health services should be organized not only for the diagnosis and provision of multidrug therapy, but for all aspects involving the disease.

Key words Leprosy, Prevention, Disability, Documents
\end{abstract}

Resumo As orientações para prevenção de incapacidades físicas por hanseníase vêm ganhando destaque no controle da doença para além da poliquimioterapia. O objetivo deste artigo é descrever as mudanças, de forma cronológica, nas orientações técnicas para prevenção das incapacidades físicas por hanseníase no Brasil. Trata-se de relato histórico baseado em documentos oficiais dos órgãos de saúde brasileiros. Foram incluidos todos os decretos, portarias e manuais que fizeram menção à prevenção de incapacidades, publicados entre 1962 e 2016. Os documentos estabeleceram os critérios clínicos, técnicos, assistenciais, emocionais e sociais, de direcionamento das ações de prevenção nas instituições de saúde. As publicações foram coordenadas pelo Ministério da Saúde e adequadas ao longo dos anos sob o ponto de vista conceitual, político, estratégico e assistencial. As orientações abrangeram, principalmente, as atividades de avaliação neurológica e do grau de incapacidade física, técnicas de prevenção de incapacidades e autocuidado. As incapacidades físicas refletem a qualidade do acesso ao diagnóstico, do acompanhamento dos casos durante o tratamento e pós-alta por cura. Os serviços de saúde deverão ser organizados não apenas para o diagnóstico $e$ oferta da poliquimioterapia, mas para todos os aspectos que envolvem a doença.

Palavras-chave Hanseníase, Prevenção, Incapacidade, Documentos 


\section{Introdução}

A hanseníase classificada como doença negligenciada com potencial de eliminação, destaca-se em virtude do número de pessoas infectadas, do estigma associado e, especialmente, pelo potencial em produzir incapacidades físicas (IF) em consequência do comprometimento neural ${ }^{1,2}$. Uma das características da doença é o tropismo do M. leprae por nervos periféricos, resultando em maior comprometimento dos nervos dos olhos, mãos e pés ${ }^{3}$. Durante a evolução clínica, o dano neural pode ocorrer de forma lenta e silenciosa, com diminuição da sensibilidade térmica, da progressiva perda da sensibilidade dolorosa, seguida da tátil ${ }^{4}$.

O comprometimento neural é um aspecto grave da hanseníase devido ao potencial incapacitante, atualmente mensurado em graus de 0 a 2 (zero a dois); 0 para ausência IF e 2 para IF mais acentuadas. As deficiências físicas provocadas pela doença podem variar de perda de sensibilidade até incapacidades visíveis nas mãos, pés e olhos $^{4}$.

Em 2016, a Organização Mundial de Saúde (OMS), lançou a Estratégia Global 2016-2020, com o propósito de priorizar a detecção precoce da hanseníase e o tratamento imediato, de modo a evitar as incapacidades e reduzir a transmissão. A estratégia também propicia maior visibilidade e relevância aos aspectos humanos e sociais. Ressalta a necessidade de redução do estigma e a promoção da inclusão social, fatores que afetam o controle da doença, principalmente em áreas essenciais de intervenção. Um dos focos da Estratégia Global é voltado para as IF, para o qual recomenda modificações operacionais estratégicas. Como novas metas são apresentadas a ausência de IF entre os casos novos em menores de 15 anos e menos de 1 caso com grau 2 de incapacidade por 1 milhão de habitantes ${ }^{5}$. Essa iniciativa ocorre em resposta ao número crescente de casos com grau 2 de IF em sentido inverso à redução da detecção global da doença ${ }^{6}$. No Brasil, a proporção de casos de hanseníase com grau 2 de IF no diagnóstico foi de 7,5\% em 2015 , enquanto a proporção média mundial foi de $6,7 \%{ }^{6}$.

O reconhecimento de incapacidades é complexo porque afeta além do corpo físico, o convívio familiar e social ${ }^{8}$. Estudos mostram que as consequências, em decorrência das IF por hanseníase na vida das pessoas incluem: limitações para a realização de atividades cotidianas, diminuição da capacidade de trabalho ${ }^{9-12}$, baixo nível de autoeficácia, além das restrições de participa- ção social por medo e vergonha que os afetados sentem de sua aparência ${ }^{10,13}$.

Segundo Arvello ${ }^{14}$ a necessidade de prevenção de incapacidade física por hanseníase teve início em 1947, com o Dr. Paul Brand, que mostrou que as úlceras e as mutilações não eram consequências inevitáveis da doença, podendo ser prevenidas e tratadas por meio da educação do doente, preconização de massagens, exercícios e uso de férulas. Na década de 1960, a OMS reconhecendo a possibilidade e a relevância de controle do comprometimento neural, determinou como prioridade a prevenção de incapacidades físicas ${ }^{15}$. Para tanto, foi elaborado o primeiro instrumento de avaliação de incapacidades, baseado na proposta formulada por Bechelli e Dominguez de 1966. O formulário determinava a avaliação de mãos, pés e olhos, compreendidas como regiões com maior grau de comprometimento, de diagnóstico simples e que provocavam mais restrições nas atividades cotidianas ${ }^{5,16}$.

No Brasil, as orientações oficiais para a prevenção das incapacidades físicas por hanseníase tiveram início na década de 1962, com a divulgação do decreto intitulado "Normas Técnicas Especiais para o Combate à Lepra no País"17.

Após a publicação do primeiro decreto relativo ao tema, os órgãos de saúde brasileiros organizaram estratégias de controle do comprometimento neural. Uma das ações estabelecidas foi a padronização das orientações de prevenção de incapacidades em 1977, por meio da edição e distribuição de documentos impressos, portarias e manuais, contendo as orientações detalhadas. Ao longo dos anos, esses documentos foram adaptados e implementados com instrumentos que incluem os formulários de avaliação neurológica e do grau de $\mathrm{IF}^{4}$.

Sabendo-se do protagonismo em que as metas relacionadas às incapacidades físicas por hanseníase foram apresentadas na atual Estratégia Global, este estudo tem como objetivo descrever as mudanças, de forma cronológica, nas orientações técnicas para a prevenção das incapacidades físicas por hanseníase no Brasil.

\section{Método}

Estudo documental sobre as orientações técnicas para a prevenção de IF por hanseníase no Brasil, por meio de revisão histórica em linha do tempo. Foram revisados documentos de domínio público e de autoria dos órgãos de saúde. A busca foi realizada via web na Biblioteca Virtual em Saúde 
(BVS) do MS, na Secretária de Vigilância em Saúde (SVS) do MS, por meio da página www.saude. gov.br/svs, nos sites das Secretarias de Saúde dos estados e municípios, bem como, nos arquivos da Biblioteca do Instituto Lauro de Souza Lima (ILSL) em Bauru-SP, referência para a Organização Pan-Americana de Saúde para hanseníase na América Latina. Os websites das secretarias estaduais e municipais de saúde foram consultados na busca de documentos oficiais que, eventualmente, poderiam não estar disponíveis na BVS. Como descritores foram utilizadas as palavras decretos, instruções normativas, manuais e portarias, combinadas com os termos incapacidade e/ou sequela por hanseníase, independente da data de publicação. Foram considerados todos os documentos normativos/instrutivos sobre prevenção de incapacidades por hanseníase de autoria do Ministério da Saúde do Brasil, ou órgão oficial equivalente.

A análise dos documentos foi realizada em etapas. Primeiramente, avaliou-se os documentos quanto à espécie: decreto, portaria e manual. $\mathrm{Na}$ sequência, os documentos foram examinados de forma a identificar alterações nas orientações sobre prevenção de incapacidades físicas por hanseníase. Tais alterações quando identificadas foram apresentadas nos resultados por meio de figura e quadros organizados cronologicamente.

\section{Resultados}

A Figura 1 apresenta os documentos que definem as ações relacionadas à prevenção de IF na assistência à pacientes de hanseníase. Oficialmente no Brasil, o primeiro documento publicado foi o Decreto-lei no 968, de 07 de maio de 1962, da Presidência da República intitulado "Normas Técnicas Especiais Relativa ao Combate à Lepra no País". Quatorze anos depois, a Divisão Nacional de Dermatologia Sanitária do então Ministério da Saúde, publicou a Portaria no 165 , de 14 de maio de 1976 que estabeleceu a "Política de Controle da Hanseníase". No ano seguinte, foi divulgado o primeiro "Manual de Prevenção e Tratamento das Incapacidades Físicas, Mediante Técnicas Simples", de autoria da Divisão Nacional de Dermatologia Sanitária. Em 1984 foi publicado, pelo MS, o "Guia para Controle da Hanseníase" e nos anos de 1997, 2001 e 2008 o MS publicou edições reformuladas do "Manual de Prevenção de Incapacidades”.

A partir do último manual publicado em 2008, as orientações foram realizadas por meio de portarias de autoria do MS. A Portaria no 125 de 26 de março de 2009 "Define Ações de Controle da Hanseníase”. No ano seguinte a Portaria no 3.125 de 07 de outubro de 2010 "Aprova as Diretrizes para Vigilância, Atenção e Controle da Hanseníase" e a Portaria no 594 de 29 de outubro de 2010 sobre "Atenção Integral em Hanseníase”. Em 03 de fevereiro 2016 foi publicada a Portaria no 149 que "Aprova as Diretrizes para Vigilância, Atenção e Eliminação da Hanseníase”. No mesmo ano é divulgado o manual técnico-operacional, intitulado "Diretrizes para Vigilância, Atenção e Eliminação da Hanseníase como Problema de Saúde Pública", documento que atualmente orienta a prevenção de incapacidades por hanseníase no Brasil.

O Quadro 1 mostra a sequência cronológica e as principais orientações sobre prevenção de incapacidades descritas nos documentos brasileiros. O primeiro Decreto-lei, no 968, de 07 de maio de 1962, recomendou cirurgias reparadoras como tratamento para as então denominadas "deformidades". Na Portaria no 165, de 14 de maio de 1976, pela primeira vez foi utilizado o termo "incapacidade física" e recomendou ações de prevenção de incapacidades para as instituições de saúde no Brasil. O primeiro manual de prevenção de incapacidades do MS intitulado "Manual de Prevenção e Tratamento das Incapacidades Físicas, Mediante Técnicas Simples", publicado em 1977, disponibilizou a descrição da anatomia e fisiologia das partes do corpo afetadas pela hanseníase, bem como as recomendações para o tratamento das "lesões hansênicas". Também, abordou educação em saúde, índice de incapacidades e divulgou o primeiro formulário para registro das incapacidades. No ano de 1984 foi publicado o segundo manual intitulado "Guia para Controle da Hanseníase”, incorporando orientações sobre avaliação neurológica baseada em dor espontânea, palpação dos nervos e teste de sensibilidade. Também definiu que a prevenção de incapacidades deve ser realizada mediante técnica simples, educação em saúde, massagens, modificações de calçados, férulas, adaptação dos instrumentos de trabalho, cuidados com os olhos e ações sociais. A técnica de avaliação neurológica com a utilização dos monofilamentos de Semmes Weinsten e a avaliação da força motora foram descritos no "Manual de Prevenção de Incapacidades” de 1997. A simplificação do formulário de avaliação neurológica e as orientações a respeito de autocuidados, a partir do modelo Preceding, Reinforcing, Enabling Causes in Educational Diagnosis and Evaluation (PRECEDE), que permite 


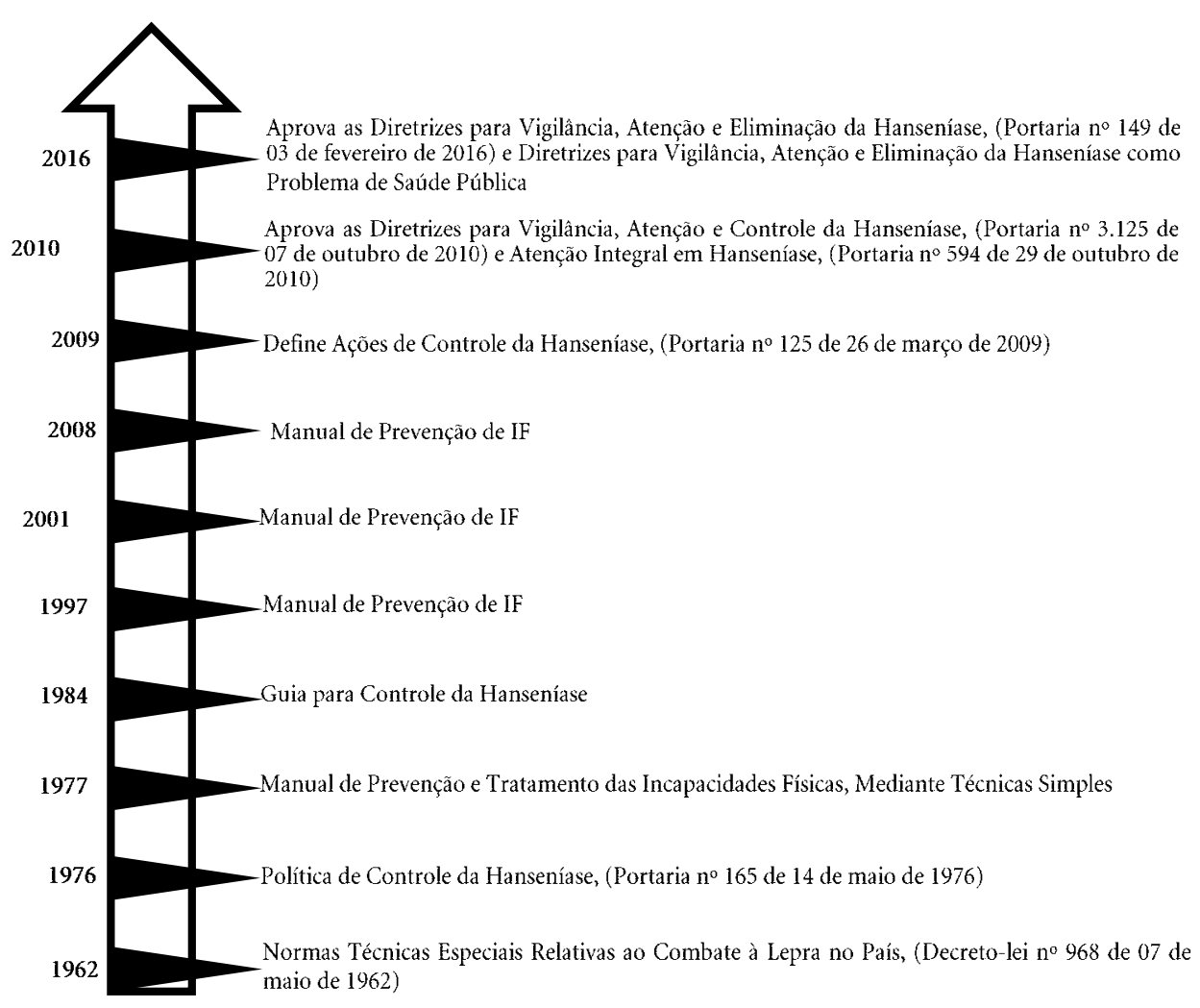

Figura 1. Ordem cronológica dos documentos publicados para orientar as ações relacionadas à incapacidade física (IF) por hanseníase no Brasil.

Fonte: Biblioteca Virtual em Saúde e arquivos do Instituto Lauro de Souza Lima.

Quadro 1. Principais orientações sobre prevenção de incapacidades físicas por hanseníase descritas nos documentos publicados pelos órgãos de saúde no Brasil no período de 1962 a 2016.

\begin{tabular}{|c|c|}
\hline Documento & Principais Orientações \\
\hline $\begin{array}{l}\text { Decreto no968 de } \\
1962\end{array}$ & revenção das deformidades pelos métodos não cirúrgicos e correção cirúrgica das deformidades \\
\hline $\begin{array}{l}\text { Portaria no165 de } \\
1976\end{array}$ & $\begin{array}{l}\text {-Determina ações de prevenção das IF entre as atividades de rotina em todos os hospitais, serviços } \\
\text { especializados e nos serviços gerais das instituições de saúde }\end{array}$ \\
\hline $\begin{array}{l}1^{\circ} \text { manual de } \\
\text { PI/1977 }\end{array}$ & $\begin{array}{l}\text {-Composto por noções de anátomo-fisiologia e do exame funcional dos membros superiores e } \\
\text { inferiores e dos olhos, por meio da inspeção, palpação e testes de força muscular } \\
\text { - Descreve o tratamento para as lesões hansênicas com calor úmido, imobilização, massagem de } \\
\text { estiramento com óleo, exercícios musculares passivos e ativos. Orienta a adaptação de instrumentos } \\
\text { de trabalhos, utensílios domésticos e palmilhas } \\
\text {-Recomenda o uso de férulas } \\
\text {-Aborda educação em saúde e indice de incapacidades e apresenta o primeiro formulário para registro } \\
\text { das IF }\end{array}$ \\
\hline $\begin{array}{l}2^{\circ} \text { manual de } \\
\mathrm{PI} / 1984\end{array}$ & $\begin{array}{l}\text {-Orienta o exame neurológio baseado em dor espontânea ou à palpação nos troncos nervosos, bem } \\
\text { como, a pesquisa de sensibilidade térmica, dolorosa e tátil, com tubo de ensaio, alfinete e mecha de } \\
\text { algodão } \\
\text {-Define as ações que compõem a prevenção de IF }\end{array}$ \\
\hline
\end{tabular}


Quadro 1. Principais orientações sobre prevenção de incapacidades físicas por hanseníase descritas nos documentos publicados pelos órgãos de saúde no Brasil no período de 1962 a 2016.

\begin{tabular}{|c|c|}
\hline Documento & Principais Orientações \\
\hline $\begin{array}{l}3^{\circ} \text { manual de } \\
\text { PI/1997 }\end{array}$ & $\begin{array}{l}\text {-Descreve a fisiopatogenia das incapacidades, especifica as alterações sensitivas, motoras e } \\
\text { autonômicas e aborda mãos e pés reacionais } \\
\text {-Determina avaliação neurológica, por meio do teste de sensibilidade e avaliação da força motora, } \\
\text { realizada no diagnóstico, na alta, com um ano e nas queixas } \\
\text {-Recomenda o uso do conjunto de monofilamentos de Semmes-Weinstein (6 monofilamentos:0.05g, } \\
0.2 \mathrm{~g}, 2 \mathrm{~g}, 4 \mathrm{~g}, 10 \mathrm{~g} \text { e } 300 \mathrm{~g} \text { ) nos pontos de avaliação de sensibilidade em mãos e pés. Para os olhos o uso } \\
\text { do fio dental (sem sabor), os testes de Schirmer, sensibilidade da córnea, avaliação da pressão ocular } \\
\text { e escala de Snellen } \\
\text {-Propõe o teste da força muscular com graduação de } 0 \text { a } 5 \text {, utilizando os termos paralisia, paresia e } \\
\text { normal e orienta a prevenção e o tratamento das IF com hidratação e lubrificação da pele, massagens } \\
\text { e exercícios musculares e o uso de férulas e palmilhas } \\
\text {-Possui roteiro para avaliação neurológica do nariz, olhos, mãos e pés, e também, formulário para } \\
\text { registro das IF }\end{array}$ \\
\hline $\begin{array}{l}4^{\circ} \text { manual de } \\
\mathrm{PI} / 2001\end{array}$ & $\begin{array}{l}\text { Discute autocuidado, com o modelo PRECED e apresenta formulário de avaliação neurológica } \\
\text { simplificada e do grau de IF } \\
\text {-Descreve a diferença entre avaliação neurológica e grau de IF }\end{array}$ \\
\hline $\begin{array}{l}5^{\circ} \text { manual de } \\
\mathrm{PI} / 2008\end{array}$ & $\begin{array}{l}\text { Aborda acolhimento, discriminação, humanização da assistência, avaliação de comunicantes, } \\
\text { autocuidado, independência e cura e apresenta o roteiro de avaliação da face, nariz, olhos e membros } \\
\text { superiores e inferiores, assim como, orienta a prevenção e o tratamento das incapacidades } \\
\text {-Recomendada o uso da ponta da caneta esferográfica, na falta do monofilamento lilás, para o teste } \\
\text { de sensibilidade de mãos e pés } \\
\text {-Os critérios de graduação da força muscular variam de } 0 \text { a } 5 \text {, e os resultados são definidos em forte, } \\
\text { diminuída e paralisada. } \\
\text {-Orienta o escore olhos, mãos e pés (EHF) e apresenta a escala de "Screening of Activity Limitation and } \\
\text { Safety Awareness" (SALSA) e Escala de participação (EP) }\end{array}$ \\
\hline $\begin{array}{l}\text { Portaria } n^{\circ} \\
125 / 2009\end{array}$ & $\begin{array}{l}\text { Orienta a assistência ao paciente em todas as instâncias e em diferentes níveis de complexidade, } \\
\text { de acordo com os princípios do SUS e define a avaliação neurológica e o grau de IF como } \\
\text { imprescindíveis no diagnóstico e nos estados reacionais } \\
\text {-Não recomenda o uso da caneta esferográfica no exame neurológico e estabelece o diagnóstico } \\
\text { precoce como meio de prevenção das incapacidades } \\
\text {-As ações de prevenção de incapacidades são recomendadas para todos os pacientes e a } \\
\text { avaliação neurológica deve ser realizada no início do tratamento, a cada três meses, nas queixas, } \\
\text { periodicamente em pacientes em uso de corticoides, com reações e/ou neurites, na alta e no pós- } \\
\text { operatório } \\
\text {-Preconiza o acompanhamento no pós-alta por cura, referência e contrarreferência e nas práticas de } \\
\text { educação em saúde } \\
\text {-Define como indicadores epidemiológicos e operacionais: proporção de casos de hanseníase com grau } \\
2 \text { de IF no momento do diagnóstico, entre os casos novos detectados e avaliados no ano e proporção } \\
\text { de casos de hanseníase com grau } 2 \text { de IF, entre os casos avaliados no momento da alta por cura }\end{array}$ \\
\hline $\begin{array}{l}\text { Portaria } \\
\text { no3125/2010 }\end{array}$ & $\begin{array}{l}\text { Recomenda a realização das técnicas de autocuidados no domicílio e em outros ambientes e auto } \\
\text { inspeção diária } \\
\text {-Define como ação prioritária da Programação das Ações de Vigilância em Saúde, (PAVS), a avaliação } \\
\text { no diagnóstico do grau de IF dos casos novos de hanseníase e dos curados no ano de avaliação } \\
\text {-Acrescenta o Coeficiente de grau } 2 \text { de IF dos casos novos por } 100.000 \text { habitantes no momento do } \\
\text { diagnóstico como indicador de monitoramento e avaliação da hanseníase }\end{array}$ \\
\hline $\begin{array}{l}\text { Portaria } n^{\circ} \\
594 / 2010\end{array}$ & $\begin{array}{l}\text { Estabelece a integralidade da assistência, nos diferentes níveis de atenção e define serviços para } \\
\text { prevenção e tratamento das incapacidades incluindo: prevenção de incapacidades, com técnicas } \\
\text { simples e autocuidado apoiado pela equipe e atendimento pré e pós-operatório, procedimentos } \\
\text { cirúrgicos e acesso a órteses, palmilhas e calçados adaptados. }\end{array}$ \\
\hline $\begin{array}{l}\text { Manual técnico- } \\
\text { operacional/2016 }\end{array}$ & $\begin{array}{l}\text { A PI inclui conjunto de medidas visando evitar danos físicos, emocionais e socioeconômicos e } \\
\text { complicações dos danos existentes e devem ser realizadas nas unidades de saúde, mediante utilização } \\
\text { de técnicas simples (educação em saúde, exercícios preventivos, adaptações de calçados, férulas, } \\
\text { adaptações de instrumentos de trabalho e cuidados com os olhos) } \\
\text {-Os casos de IF que requererem técnicas complexas devem ser encaminhados aos serviços } \\
\text { especializados ou serviços gerais de reabilitação }\end{array}$ \\
\hline
\end{tabular}

Fonte: Biblioteca Virtual em Saúde e arquivos do Instituto Lauro de Souza Lima. 
identificar fatores que facilitam ou impedem a realização adequada dos autocuidados, foram definidas no "Manual de Prevenção de Incapacidades" de 2001.

Questões como acolhimento, discriminação, humanização da assistência, avaliação de comunicantes e independência pessoal foram introduzidas no manual de 2008, intitulado "Manual de Prevenção de Incapacidades". Também foi abordado o escore olhos, mãos e pés (EHF) como avaliação alternativa do grau máximo de incapacidade da OMS, com o propósito de resumir os dados sobre as deficiências. A avaliação EHF permite determinar o grau máximo para cada um dos seis locais do corpo (olhos, mãos e pés) cujo resultado é a somatória do grau de IF de cada um dos locais avaliados, podendo variar de 0 a 12. Apresentou também a escala de Screening of Activity Limitation and Safety Awareness (SALSA) e a Escala de Participação (EP). Estes instrumentos permitem, respectivamente, medir limitação de atividade, consciência de risco e restrições à participação social.

A Portaria no 125, de 26 de março de 2009, estabeleceu a assistência ao paciente de hanseníase em todas as instâncias e nos diferentes níveis de complexidade, de acordo com os princípios do Sistema Único de Saúde (SUS). Definiu o diagnóstico precoce da doença como ação capaz de prevenir incapacidades e determinou a inclusão da proporção de casos com grau 2 (dois) de IF como indicador de monitoramento e avaliação da hanseníase.

Na Portaria no 3.125 , de 07 de outubro de 2010, foram definidas as técnicas de autocuidados no domicílio e em outros ambientes, assim como, manteve a orientação para a avaliação do grau de IF dos casos novos de hanseníase no diagnóstico e na saída por encerramento da poliquimioterapia $(\mathrm{PQT})$, preconizada desde o manual de 1977. O conceito de integralidade da assistência à saúde foi estabelecido na Portaria no 594, de 29 de outubro de 2010, que também definiu serviços para prevenção das incapacidades. O Manual Técnico-Operacional, intitulado "Diretrizes para Vigilância, Atenção e Eliminação da Hanseníase como Problema de Saúde Pública", de 2016, estabeleceu um conjunto de medidas, com a finalidade de evitar a ocorrência de danos físicos, emocionais e socioeconômicos aos pacientes de hanseníase. Para os casos de incapacidades de maior complexidade orientou encaminhamento para aos serviços especializados e de reabilitação.

O Quadro 2 apresenta as principais alterações entre o primeiro formulário de avaliação neuro- lógica e a última e atual versão publicada depois de quase vinte anos. O primeiro formulário padronizado pelo MS para pacientes de hanseníase foi recomendado no manual de IF de 1997. A versão reformulada foi publicada nos manuais de 2008 e 2016. Entre as alterações destaca-se a simplificação do formulário em relação ao conteúdo e a quantidade de páginas. Os registros ficaram restritos a um único formulário para a avaliação dos seguimentos nariz, olhos e membros superiores e inferiores. Na versão publicada em 1997, a avaliação era realizada em formulários específicos para cada seguimento. Quanto às técnicas utilizadas, mantiveram os mesmos procedimentos, exceto pela exclusão do teste Schimer para os olhos e de sensibilidade para a região dorsal das mãos e pés. Outra alteração foi o aumento do número de avaliações neurológicas, recomendadas de modo mais precoce em relação ao diagnóstico e em intervalo de tempo menor. Também foi instituída a avaliação neurológica no acompanhamento pós-operatório de reparação da IF. O formulário utilizado atualmente foi publicado em 2016 no manual "Diretrizes para Vigilância, Atenção e Eliminação da Hanseníase como Problema de Saúde Pública".

O Quadro 3 mostra a descrição dos formulários de avaliação do grau de IF, padronizados e divulgados nos documentos oficiais do MS. O primeiro formulário foi publicado no manual de incapacidade física em 1977. A mesma versão, sem alterações, foi novamente publicada no manual de 1984. Os formulários foram reformulados, em 1997 e 2001 em relação à medida do grau do IF, agrupamento de incapacidades, uso de novas terminologias e do registro da ausência de incapacidades. O formulário mais recente foi publicado em 2016, com maior detalhamento dos critérios para determinação do grau de IF. De forma geral, os primeiros formulários publicados em 1977 e 1984 eram restritos a pacientes com incapacidades instaladas, enquanto os mais recentes permitem também a avaliação de pacientes sem danos neurais.

\section{Discussão}

Os documentos oficiais que estabeleceram a prevenção de incapacidades por hanseníase nas instituições de saúde brasileiras apresentaram estrutura e abordagem diferenciadas, porém convergiram nas orientações sobre o controle do dano neural, priorizando estratégias para evitar e/ou minimizar as incapacidades. 
Quadro 2. Comparação entre os formulários de avaliação neurológica, padronizados pelo Ministério da Saúde, nos anos de 1997 e 2016.

\begin{tabular}{|c|c|c|}
\hline Documento & Manual de 1997 & Manual de 2016 \\
\hline Denominação & $\begin{array}{l}\text {-Prevenção de Incapacidades físicas na } \\
\text { hanseníase: Roteiro de Avalição }\end{array}$ & -Avaliação Neurológica simplificada \\
\hline Descrição & $\begin{array}{l}\text {-Formulários separados: nariz e olhos; membros } \\
\text { superiores e membros inferiores. Em cada } \\
\text { segmento é determinado o grau de IF } \\
\text {-Recomenda avaliação: no diagnóstico, decorrer } \\
\text { do tratamento, intervalos de } 6 \text { meses, com } \\
\text { maior frequência durante neurites e reações, na } \\
\text { apresentação de queixas e no ato da alta }\end{array}$ & $\begin{array}{l}\text {-Formulário único para avaliação } \\
\text { dos segmentos nariz, olhos, membros } \\
\text { superiores e membros inferiores } \\
\text {-Recomenda avaliação: no início do } \\
\text { tratamento, a cada } 3 \text { meses, nas queixas, } \\
\text { periodicamente em pacientes com reação } \\
\text { e neurites, na alta e no acompanhamento } \\
\text { pós-operatório de descompressão neural }\end{array}$ \\
\hline Técnica/material & $\begin{array}{l}\text {-Nariz e olhos: Entrevista, inspeção, exploração } \\
\text { da força muscular e exame ocular, com o teste de } \\
\text { Schimer e Snellen } \\
\text {-Membros superiores e inferiores: Entrevista, } \\
\text { inspeção, palpação dos nervos, exploração da } \\
\text { mobilidade articular, força muscular e pesquisa de } \\
\text { sensibilidade com estesiômetro. Examina-se mãos, } \\
\text { região dorsal e palmar e pés, dorsal e plantar } \\
\text {-O resultado é registrado em números de } 0 \text { à 6, } \\
\text { em que cada um representa um monofilamento. } \\
\text { Utiliza ilustrações para registro das alterações } \\
\text { (calosidades, ferimento, cicatriz e reabsorção) }\end{array}$ & $\begin{array}{l}\text {-Manteve-se a técnica/materiais, exceto: } \\
\text { retirada do teste de Schimer para os } \\
\text { olhos e de sensibilidade para região } \\
\text { dorsal das mãos e pés } \\
\text {-Utiliza "sente" (v) ou "não-sente" (X), } \\
\text { como terminologia para as alterações } \\
\text { ou segue as cores dos monofilamentos } \\
\text { para registrar as alterações }\end{array}$ \\
\hline Avaliação & $\begin{array}{l}\text {-Nariz: condições da pele, mucosa e septo nasal } \\
\text {-Olhos: avaliação das pálpebras, conjuntiva, } \\
\text { córnea, pupila e cristaliano } \\
\text {-Membros superiores: avaliação das articulações } \\
\text { interfalangeanas e do } 1^{\circ} \text { espaço dorsal (sem } \\
\text { problemas, garra móvel ou rígida); palpação dos } \\
\text { nervos radial, radial cutâneo, ulnar e mediano } \\
\text { (sem problema, dor, espessado, tinel/choque, } \\
\text { fibroso e nódulos); força muscular do nervo radial } \\
\text { (extensão do punho), ulnar (abdução do } 2^{\circ} \text { e } 5^{\circ} \\
\text { dedo e posição intrínseca do } 5^{\circ} \text { ) e nervo mediano } \\
\text { (abdução do polegar) } \\
\text {-Força muscular registrada em forte, diminuída, } \\
\text { paralisia ou graduar de } 0 \text { à } 5 \\
\text {-Membros Inferiores: Inspeção (ressecamento e } \\
\text { pé caído). Avaliação da articulações tíbio-társica, } \\
\text { articulações interfalangeanas ( halux, proximal } \\
\text { do 2º ao } 5^{\circ} \text { artelho); palpação dos nervos fibular } \\
\text { comum e tibial posterior, (sem problema, dor, } \\
\text { espessado, tinel/choque, fibroso e nódulos); a } \\
\text { força muscular do nervo fibular comum (extensão } \\
\text { do hálux e dos artelhos, dorsiflexão é registrada } \\
\text { em forte, diminuída, paralisia ou graduar de } 0 \text { à } 5 \\
\text {-Registra a adaptação de calçados }\end{array}$ & $\begin{array}{l}\text {-Nariz: ressecamento, ferida e } \\
\text { perfuração de septo } \\
\text {-Olhos: Deixou-se de registrar a paresia } \\
\text { nas pálpebras, a conjuntiva, a pupila e a } \\
\text { pressão intraocular } \\
\text {-Membros superiores: Não } \\
\text { registra avaliação das articulações } \\
\text { interfalangeanas e palpação do nervo } \\
\text { radial cutâneo. } \\
\text {-Legenda passa a ser nervos normais } \\
\text { (N), espessado (E) e dor (D) } \\
\text {-Na avaliação da força muscular } \\
\text { acrescentou elevação do punho para } \\
\text { o nervo radial e elevar o polegar no } \\
\text { mediano, para o nervo ulnar, abrir dedo } \\
\text { mínimo e a abdução do } 5^{\circ} \text { dedo } \\
\text {-Membros Inferiores: Não registra } \\
\text { inspeção e avalição das articulações } \\
\text { tíbio-társica e interfalangeanas } \\
\text {-Na força muscular acrescentou elevar } \\
\text { o hálux e o pé, não avalia extensão dos } \\
\text { artelhos e eversão do pé }\end{array}$ \\
\hline
\end{tabular}

Fonte: Biblioteca Virtual em Saúde e arquivos do Instituto Lauro de Souza Lima.

A análise documental mostra o aprimoramento das ações de prevenção das sequelas da hanseníase, desde a primeira normatização em 1962. As publicações foram atualizadas ao longo dos anos, com a inclusão de técnicas e ações propostas por organizações como a OMS e OPAS/ $\mathrm{OMS}^{4,18}$. A implementação ocorreu de forma gradativa e foi organizada conforme a estruturação 
Quadro 3. Descrição dos formulários de avaliação do grau de incapacidade física, por hanseníase, publicados no Brasil no período de 1977 a 2016.

\begin{tabular}{|c|c|c|}
\hline Documento & Descrição & Graduação \\
\hline $\begin{array}{l}\text { Formulário/1977 } \\
\text { Formulário/1984 }\end{array}$ & $\begin{array}{l}\text {-Graduação das } \\
\text { alterações varia de } \\
1 \text { a } 3 \\
\text {-Avaliação da laringe, } \\
\text { nariz e paralisia facial } \\
\text {-Registra uma } \\
\text { avaliação, determina } \\
\text { a soma dos graus, } \\
\text { maior grau e índice de } \\
\text { incapacidade }\end{array}$ & $\begin{array}{l}\text { 1-Anestesia nas mãos ou pés e/ou conjuntivite nos olhos } \\
\text { 2-Maõs: úlceras, lesões traumáticas, garra móvel, reabsorção } \\
\text { discreta } \\
\text {-Pés: úlceras tróficas, garra dos artelhos, pé caído e reabsorção } \\
\text { discreta } \\
\text {-Olhos: conjuntivite, lagoftalmo, irite ou ceratite, diminuição da } \\
\text { acuidade visual } \\
\text { 3-Mãos: mão caída, articulações anquilosadas e reabsorção intensa } \\
\text {-Pés: contratura e reabsorção intensa } \\
\text {-Olhos: acentuada diminuição da visão e cegueira }\end{array}$ \\
\hline Formulário/1997 & $\begin{array}{l}\text {-Graduação das } \\
\text { alterações varia de } \\
0 \text { a } 3 \\
\text {-Avaliação da laringe, } \\
\text { nariz e paralisia facial } \\
\text {-Registra uma } \\
\text { avaliação, determina } \\
\text { a soma dos graus, } \\
\text { maior grau e índice de } \\
\text { incapacidade }\end{array}$ & $\begin{array}{l}\text { 0-Sem problemas nas mãos, pés e olhos devido a hanseníase } \\
\text { 1-Anestesia nos olhos, nas mãos ou nos pés } \\
\text { 2-Maõs: úlceras, lesões traumáticas, garra móvel, reabsorção } \\
\text { discreta } \\
\text {-Pés:úlceras tróficas, garra dos artelhos, pé caído e reabsorção } \\
\text { discreta } \\
\text {-Olhos: lagoftalmo ou ectrópio, triquíase e opacidade corneana } \\
\text { 3-Mãos: mão caída, articulações anquilosadas e reabsorção intensa } \\
\text {-Pés: contratura e reabsorção intensa } \\
\text {-Olhos: acuidade visual menor que } 0,1 \text { ou não conta dedos a } 6 \mathrm{~m}\end{array}$ \\
\hline Formulário/2001 & $\begin{array}{l}\text {-Graduação das } \\
\text { alterações varia de } \\
0 \text { a } 2 \\
\text {-Registra duas } \\
\text { avaliações, no } \\
\text { diagnóstico da } \\
\text { doença e na alta do } \\
\text { tratamento } \\
\text {-Determina o maior } \\
\text { grau nos olhos, mãos } \\
\text { e pés }\end{array}$ & $\begin{array}{l}\text { 0-Sem problemas nos olhos, mãos e pés decorrentes da } \\
\text { hanseníase } \\
\text { 1-Diminuição ou perda da sensibilidade nos olhos, } \\
\text { mãos e pés (não sente } 2 \mathrm{~g} \text { ou o toque da caneta) } \\
\text { 2-Olhos: lagoftalmo ou ectrópio, triquíase, opacidade } \\
\text { corneana e acuidade visual menor que } 0,1 \text { ou não cl } \\
\text { conta dedos a } 6 \mathrm{~m} \\
\text {-Mãos: lesões tróficas e/ou traumáticas, garras, } \\
\text { reabsorção e mão caída } \\
\text {-Pés: lesões tróficas e/ou traumáticas, garras, reabsorção, pé caído } \\
\text { e contratura de tornozelo }\end{array}$ \\
\hline Formulário/2016 & $\begin{array}{l}\text {-Graduação das } \\
\text { alterações varia de } \\
0 \text { a } 2 \\
\text {-Registra as avaliações } \\
\text { realizadas no } \\
\text { diagnóstico e na alta } \\
\text {-Determina o maior } \\
\text { grau e a soma OMP } \\
\text { (olhos, mãos e pés) }\end{array}$ & $\begin{array}{l}\text { 0-Olhos: Força muscular das pálpebras e sensibilidade } \\
\text { da córnea preservadas e/ou conta dedos a } 6 \text { metros ou } \\
\text { acuidade visual > =0,1 ou 6:60 } \\
\text {-Mãos e pés: força muscular preservadas e } \\
\text { sensibilidade palmar e plantar (sente o } \\
\text { monofilamento } 2 \mathrm{~g} \text {, lilás, ou o toque da ponta de } \\
\text { caneta esferográfica } \\
\text { 1-Olhos: diminuição da força muscular das pálpebras, } \\
\text { sem deficiências visíveis e/ou diminuição da } \\
\text { sensibilidade da córnea (resposta demorada ou } \\
\text { ausente ao toque do fio dental ou } \\
\text { diminuição/ausência do piscar) } \\
\text {-Mãos e pés: diminuição da força muscular das mãos } \\
\text { sem deficiências visíveis e/ou alteração de } \\
\text { sensibilidade, palmar e/ou plantar, (não sente } 2 \mathrm{~g}, \\
\text { lilás, ou o toque da ponta da caneta esferográfica) } \\
\text { 2- Olhos: deficiências visíveis causada pela hanseníase } \\
\text { (lagoftalmo, ectrópio, entrópio, triquíase, opacidade } \\
\text { corneana central, iridociclite e/ou não conta dedos a } \\
\text { 6m ou acuidade visual menor que } 0,1 \text { ou } 6: 60 \\
\text {-Mãos e pés: garras, reabsorção óssea, atrofia } \\
\text { muscular, mão/pé caído, contratura e ferida } \\
\text { Pés: lesões tróficas e/ou traumáticas, garras, reabsorção, pé caído e } \\
\text { contratura de tornozelo }\end{array}$ \\
\hline
\end{tabular}


do sistema de saúde brasileiro, incorporando os avanços científicos no diagnóstico, tratamento e acompanhamento do comprometimento neural em decorrência da hanseníase $e^{4}$.

Em 1962 foi estabelecida a necessidade de acompanhamento do comprometimento neural, instituindo a obrigatoriedade do tratamento com ênfase na prevenção das "deformidades"17. Inicialmente, merece destaque o termo "deformidade" como referência às sequelas provocadas pela hanseníase. O uso da expressão era comum naquele período e pode estar relacionado ao número de casos com sequelas visíveis. Estudos realizados entre 1920 e 1970 mostraram que casos com deformidades representavam cerca de 30 a 70 por cento, do total de doentes ${ }^{19-22}$. Também, é observada a inespecificidade do documento, pois determinava a necessidade de adoção de ações preventivas no atendimento aos pacientes por meio do método não cirúrgico, sem, no entanto, definir como e quem deveria realizar tais ativida$\operatorname{des}^{17}$. Comumente, na década de 1960 , as orientações e os treinamentos, para acompanhamento e tratamento das sequelas, eram realizados por especialistas em hanseníase. Estes capacitavam um grupo de pessoas da área da saúde, responsáveis pelo atendimento aos pacientes de hanseníase, tornando-os multiplicadores e encarregados pela orientação dos demais profissionais dos centros especializados que concentravam os atendimentos na época ${ }^{23}$. Ressalta-se que neste período, a política vigente para assistência aos pacientes de hanseníase era baseada no isolamento compulsório, com a internação dos doentes em colônias denominadas leprosários e afastamento dos contatos familiares e sociais ${ }^{24}$.

A inclusão de ações, com enfoque na prevenção de incapacidades, entre as atividades de rotina das instituições de saúde foi determinada em portaria no ano de 1976. Mas, assim como na portaria de 1962, as ações a serem desenvolvidas não foram descritas no documento. Contudo, foi estabelecido que a função de orientar a implantação de atividades preventivas ficaria a cargo da Divisão de Dermatologia Sanitária (DNDS/MS). Provavelmente, o treinamento não abrangia todos os profissionais encarregados de prestar assistência aos doentes, devido ao extenso território nacional, a organização da estrutura de saúde pública da época, a demanda crescente por assistência e as dificuldades de transporte e comunicação. Mas, pode ser considerado um avanço a prevenção como estratégia para evitar as sequelas da doença, difundindo o conceito de que as deformidades eram evitáveis ${ }^{4,25}$. A portaria $n^{\circ} 165$, de 1976, se destaca historicamente por estabelecer a instituição do regime de atendimento ambulatorial para assistência aos doentes, o emprego do termo "hanseníase" e o banimento da terminologia "lepra" e seus derivados ${ }^{25}$.

Com a publicação das primeiras portarias e diante da necessidade de orientar de forma padronizada, prática e acessível os profissionais responsáveis pela assistência de pacientes com hanseníase em todo país, em 1977 o MS publicou o primeiro manual de IF. Documento amplo e ilustrado, que aborda a anatomia e a fisiologia das áreas do corpo atingidas pela doença, técnicas simples de prevenção de incapacidade, tratamento das sequelas, além de preconizar o roteiro para a realização da avaliação física, a pesquisa de sensibilidade e o teste de força muscular. Ainda, de forma inovadora, traz entre seus anexos, o primeiro formulário de avaliação do grau de IF, com cálculo de um índice de incapacidade, como forma de quantificar gradualmente o agravo. Pela primeira vez destacou a doença como enfermidade neurológica, altamente incapacitante, em consequência do comprometimento neural, que pode ser evitado e tratado por técnicas simples, de fácil aplicação, compatível com as atividades de rotina das unidades de saúde pública ${ }^{4,26}$. Além disso, abordou a educação em saúde como estratégia fundamental para a prevenção de incapacidades, mas ressaltou que esta não depende somente dos profissionais de saúde, mas, também, da mudança de postura do doente, que precisa adquirir conhecimento sobre o assunto e modificar atitudes e práticas ${ }^{26,27}$. Esse manual foi elaborado a partir das orientações do professor José de Jesús Arvelo, que naquele período era consultor OPAS e da $\mathrm{OMS}^{26}$.

Após o primeiro manual, foi publicado o segundo, que além de abordar os conteúdos da edição anterior, apresentou novas orientações. Ressalta-se a especificação dos componentes da PI (Prevenção de Incapacidades) e o detalhamento das ações de prevenção com a finalidade de orientar os profissionais de saúde, bem como, informar que ações simples e de fácil aplicação em todas as instituições de saúde podem evitar e/ou minimizar as IF. Orientou à necessidade da realização do exame neurológico como instrumento capaz de identificar precocemente o dano neural e consequentemente evitar as sequelas mais severas da doença. $\mathrm{O}$ resultado do exame baseia-se, principalmente, na resposta do paciente à palpação dos nervos ou à verbalização espontânea de dor muscular. No entanto, a ausência de utilização de instrumentos no procedimento de 
avaliação neurológica e o resultado centralizado na manifestação verbal do paciente parece ter fragilizado a técnica, pois o resultado poderia ser comprometido por ausência de resposta ou até mesmo respostas incorretas ${ }^{18}$.

Observada a necessidade de aprimoramento da técnica de diagnóstico do dano neural, o MS publicou o terceiro manual em 1997, cujo enfoque foi avaliação neurológica. Especificou o tema, descriminou desde questões anátomofisiológicas até o diagnóstico das incapacidades, sem deixar de abordar aspectos importantes para o dano neural, como a reação hansênica, conteúdo abordado desde o manual de IF de $1977^{26}$. Nesta edição foi apresentado o monofilamento de Semmes Weinsten, como material apropriado para realização do teste de sensibilidade. O uso do estesiômetro permite maior precisão ao teste, o monitoramento da evolução da lesão nervosa periférica e a quantificação do grau de perda sensitiva em consequência da hanseníase $e^{4}$. Também foi disponibilizado o formulário de avaliação neurológica e pré-determinado a realização do exame no ato do diagnóstico, na apresentação de queixas, no decorrer do tratamento em intervalos de no máximo um ano e na alta por cura. Dessa maneira, o exame pode ser realizado de forma uniforme no Brasil, além de permitir maior especificidade na avaliação neurológica, registro adequado das informações de incapacidades, criação de banco de dados e quantificação da magnitude das sequelas da doençą, ${ }^{4,28}$. A incorporação do uso dos monofilamentos e do formulário possibilitaram o registro adequado dos dados, que podem ser utilizado como parâmetro para o tratamento das incapacidades dos indivíduos ${ }^{29,30}$.

A cada manual foram preconizadas orientações inéditas. No manual publicado em 2001, foi discutida a necessidade da participação do paciente na prevenção de IF, na forma de autocuidado, que atualmente é uma das orientações de maior destaque na prevenção de IF5. Definido como procedimentos, técnicas e exercícios, que o doente, devidamente orientado, pode realizar regularmente no seu domicílio e em outros ambientes ${ }^{4,31}$. Segundo o Ministério da Saúde (2016) a prática do autocuidado permite compartilhar responsabilidades e contribui para o diagnóstico e o tratamento precoce do dano neural. Entretanto, a incorporação de práticas de autocuidados, na assistência de pacientes de hanseníase depende, primeiramente, da ampliação da rede de atenção e da capacitação dos profissionais de saúde para um atendimento qualificado e humanizado $^{29,32}$. Por outro lado, há também a neces- sidade do comprometimento do paciente, que enfrenta dificuldades para a incorporação de práticas de autocuidado entre as atividades da vida diária. Essas dificuldades são multicausal e complexas, estão relacionadas ao estigma, à ausência de conhecimento sobre a doença, à capacidade para o autocuidado e ao compromisso com a saúde ${ }^{33}$. Ainda nesta edição, foi apresentado o formulário de avaliação neurológica simplificado, mais compacto e específico. Foram mantidas somente questões essenciais, porém suficientes, para a avaliação neurológica da face, membros superiores e inferiores ${ }^{31,34}$.

O último manual de prevenção de IF, publicado em 2008, manteve as recomendações anteriores acerca das orientações e dos procedimentos que envolvem a técnica de prevenção de IF. Acrescentou como referência para a assistência aos pacientes os conceitos de acolhimento, discriminação, humanização, autocuidado, independência e cura da doença. Justifica que se trata de conceitos e procedimentos importantes para garantir suporte e segurança ao paciente no processo de tratamento, cura, e reabilitação ${ }^{29}$. Neste manual foram redefinidas as atividades que compõem a prevenção de incapacidade, incorporando ações de cunho assistencial, psicológico e social, de forma a assistir o indivíduo na sua integralidade. Ainda, foram apresentados os formulários das escalas Screening of Activity Limitation and Safety Awareness (SALSA) e a Escala de Participação (EP), que permitem, respectivamente, identificar dificuldades nas atividades da vida diária e na inserção social dos doentes de hanseníase. A incorporação de instrumentos de cunho social possibilita conhecer como as deficiências ou o estigma associados à doença, afetam o cotidiano das pessoas. Consequentemente, torna-se possível avaliar necessidades, monitorar progressos e analisar o impacto das intervenções de reabilitação ${ }^{29}$.

Após a publicação do manual mais recente, as orientações do MS foram realizadas por meio de portarias, exceto pelo Manual Técnico-Operacional de 2016, que apesar da denominação, não apresentou estrutura similar às publicações anteriores, principalmente em relação a procedimentos clínicos e técnicas assistenciais referentes à prevenção de incapacidades. $\mathrm{O}$ manual de 2016, assim como as Portarias no 125/2009 e $3.125 / 2010$, tem a finalidade de orientar questões de organização do serviço para a assistência a pessoas com hanseníase em todas as instâncias e nos diferentes níveis de complexidades, de acordo com os princípios do Sistema Único de Saúde 
(SUS). Visa, também, o fortalecimento das ações de vigilância epidemiológica e a promoção da saúde com base na comunicação, educação e mobilização social ${ }^{35-37}$.

A reorganização da assistência aos pacientes de hanseníase, normatizada por meio das portarias, também abrangeu as incapacidades físicas. Estabeleceu as ações de prevenção paralela ao tratamento da doença de forma rotineira e em toda a rede de atenção. Ainda, destacou a hanseníase como doença com potencial incapacitante que, portanto, deve ter garantida a atenção especializada em unidades de referência de média e alta complexidade para portadores de IF, sempre que necessário, respeitando o princípio da integralidade do SUS. Pela primeira vez preconizou recomendações para a continuidade da assistência no período pós-alta por cura, especialmente para os pacientes com graus 1 e 2 de IF, afim de diagnosticar as complicações neurais e instituir tratamento apropriado ${ }^{4,35,36}$.

Outro aspecto relevante foi a inclusão dos casos de hanseníase segundo graus de IF no momento do diagnóstico e da alta por cura como indicadores de monitoramento e avaliação da doença. Essa estratégia permite avaliar a efetividade das atividades de detecção precoce e subsidiar a programação das ações de prevenção e tratamento das IF, bem como, monitorar e medir a qualidade do atendimento nos serviços de saúde no Brasil ${ }^{4,36}$.

A evolução histórica da implantação da prevenção de incapacidades nas instituições de saúde brasileiras mostra avanços nas orientações quanto à assistência aos pacientes, sobretudo no que se refere à deteç̧ão precoce do comprometimento neural. Destacam-se dois instrumentos como essências no processo: os formulários de avaliação neurológica e os de grau de IF. O primeiro para avaliação da integridade da função neural e o segundo para determinar o grau de IF do paciente em consequência do comprometimento neural ${ }^{4}$.

Ao longo das edições dos documentos oficiais, os formulários foram readequados de acordo com as necessidades dos doentes, dos avanços no tratamento da doença, bem como, na organização do sistema de saúde brasileiro. Ocorreram mudanças nos critérios de avaliação do grau de IF e no aprimoramento da técnica de avaliação neurológica. As alterações no formulário de avaliação neurológica foram relacionadas à definição do período de realização do exame, à simplificação do formulário e ao uso do estesiômetro de Semmes Weinstein. Enquanto que para a avaliação e o registro do grau de IF as mudanças referem-se particularmente às anotações dos dados. A primeira versão dos manuais de 1977 não incluiu critérios de avaliação para doentes sem comprometimento neural. Então, em 1997 acrescentou-se o grau 0 (zero) com esta finalidade. Outra alteração, em 2001, foi a exclusão do grau 3 de IF que era utilizado para sequelas mais graves da doença. As lesões visíveis foram redefinidas e consideradas como grau $2^{24,26,31}$. A última alteração foi a elaboração de critérios específicos para determinar o grau zero em pacientes de hanseníase ${ }^{4}$.

De forma geral, os dois instrumentos foram alterados em consequência da mudança no perfil dos doentes no que diz respeito às sequelas decorrentes da doença. Houve diminuição da proporção de casos com sequelas severas e aumento da proporção daqueles com menor comprometimento, como resultado da precocidade do diagnóstico, eficácia do tratamento e da assistência ao comprometimento neural ${ }^{4,38,39}$. Contudo, a qualidade das informações obtidas com a aplicação dos formulários depende de profissionais capacitados, estrutura adequada, insumos, suporte técnico e gerência atuante nos âmbitos estaduais e municipais ${ }^{34}$.

Quanto ao conceito de prevenção de incapacidades, a partir do manual de 2008 houve importante mudança, não se restringindo mais ao aspecto físico, incluiu também os danos referentes aos aspectos emocionais, espirituais e socioeconômicos ${ }^{4,29}$. A mudança do enfoque permite envolver diferentes momentos e vivências no processo de adoecimento, potencializando as ações voltadas para prevenção ${ }^{34}$.

Neste estudo não foram apresentadas as mudanças conceituais em termos cronológicos segundo a OMS, bem como, para outros países endêmicos. Mas é presumível a influência de tais orientações sobre os documentos nacionais, principalmente porque estes são citados na introdução e nas referências de cada publicação do MS.

\section{Considerações finais}

As orientações preconizadas nos documentos oficiais, sobre prevenção de IF por hanseníase no Brasil, nos últimos 54 anos, mostram importantes avanços conceituais, políticos, estratégicos e assistenciais. Evidencia também o protagonismo e o pioneirismo do Ministério da Saúde na coordenação das ações de prevenção das incapacidades físicas por hanseníase. 
As ações recomendadas tinham pelo menos três finalidades: conhecer a carga de incapacidade atribuível à hanseníase, avaliar e implementar as ações de prevenção de incapacidades e estabelecer a prevenção de incapacidades na assistência aos pacientes dessa enfermidade. As orientações abrangiam, principalmente, as atividades de avaliação neurológica e do grau de incapacidade física, técnicas de prevenção de incapacidades e autocuidado.

\section{Colaboradores}

AR Santos participou da concepção do artigo, revisão documental, da elaboração dos resultados, da análise dos dados e redação do artigo. E Ignotti participou da análise dos dados e redação do artigo.
Sabendo-se que as incapacidades físicas refletem a qualidade do acesso ao diagnóstico, do acompanhamento dos casos durante o tratamento e pós-alta por cura, os serviços de saúde deverão ser organizados não apenas para o diagnóstico e a oferta da poliquimiotrapia, mas para todos os aspectos que envolvem a doença. Nesse sentido, é preciso transcender a prática assistencial e incorporar no programa de assistência à prevenção de incapacidades ações de cunho social, familiar e cultural.

\section{Agradecimentos}

Agradecimentos à Universidade Federal de Mato Grosso. Este artigo é produto da pesquisa da autora Aleksandra Rosendo dos Santos do Programa de Doutorado em Ciências da Saúde e será usado como parte de sua tese. 


\section{Referências}

1. World Health Organization (WHO). Global leprosystrategy 2016-2020: accelerating towards a leprosy-free world. [acessado $2018 \mathrm{Fev}$ 15]. Disponível em: http:// apps.searo.who.int/PDS_DOCS/B5233.pdf

2. Brasil. Ministério da Saúde (MS). Portal da Saúde. Hanseníase 2018. [acessado 2018 Fev 15]. Disponível em : http://portalms.saude.gov.br/saude-de-a-z/hanseniase

3. World Health Organization (WHO). Leprosy Elimination, 2018. [acessado 2018 Fev 10]. Disponível em: http://who.int/lep/disease/en/

4. Brasil. Ministério da Saúde (MS). Diretrizes para vigilância, atenção e eliminação da hanseníase como problema de saúde pública. Manual técnico-operacional. Brasília: MS; 2016.

5. Organização Mundial da Saúde (OMS). Estratégia Global para Hanseníse 2016-2020: Acelerando rumo a um mundo sem hanseníase. Manual Operacional 2016. New Delhi: OMS; 2016.

6. World Health Organization (WHO). Global leprosy update, 2015: time for action, accountability and inclusion. Wkly Epidemiol Rec 2016; 91(35):405-420.

7. Diniz D. O que é deficiência. São Paulo: Brasiliense; 2007. p. 9-10.

8. Brasil. Ministério da Saúde (MS). Portal da Saúde. Dados 2015. Situação Epidemiológica. [acessado 2018 fev15]. Disponível em: http://portalms.saude.gov.br/ saude-de-a-z/hanseniase/situacao-epidemiologica

9. Boku N, Lockwood DN, Balagon MV, Pardillo FE, Maghanoy AA, Mallari EB, Cross H. Impacts of the diagnosis of leprosy and of visible impairments amongst people affected by leprosy in Cebu, the Philippines. Lepr Rev 2010; 81, 111-120.

10. Groot RG, Van Brakel WH, Vries HJC. Social implications of leprosy in the Netherlands - stigma among ex-leprosy patients in a non-endemic setting. Lepr Rev 2011; 82(2):168-177.

11. Nardi SMT, Paschoal VDA, Zanetta DMT. Limitations in activities of people affected byleprosy after completing multidrug therapy: application of the SALSA scale. Lepr Rev 2012; 83(2):172-183.

12. Van Brakel WHV, Sihombing B, Djarir H, Beise K, Kusumawardhani L, Yulihane R, kurniasari I, kasim M, kesumaningsihKi, Wilder-Smith A. Disability in people affected by leprosy: the role of impairment, activity, social participation, stigma and discrimination. Glob Health Action 2012; 5.

13. De Castro LE, Da Cunha AJ, Fontana AP, De Castro Halfoun VL, Gomes MK. Physical disability and social participation in patients affected by leprosy after discontinuation of multidrug therapy. Lepr Rev 2014; 85(3):208-217.

14. Arvello JJ. Prevenção de Incapacidades Físicas e Reabilitação em Hanseníase. In: Uerksen E, Virmond M, organizadores. Cirurgia Reparadora e Reabilitação em Hanseníase. Bauru: Alm internacional; 1997. p. 35-47.

15. Organização Mundial da Saúde (OMS). Reunion Cientifica sobre la rehabilitacion de los leprosos. Séries de Informes Técnicos, no 221. Vellore, Índia, 1961. [acessado $2018 \mathrm{Fev}$ 08]. Disponível em: http://search. who.int/search?q=informe $+221+$ of $+1961 \& i e=u-$ tf8\&site=default_collection\&client=searo_en_r\&proxystylesheet $=$ searo_en_r\&output $=x m l \_n o \_d t d \& o$ $\mathrm{e}=$ utf8
16. Bechelli LM, Dominguez MV. Disability index for leprosy patients. Bull Wld Hlth Org 1971; 44(5):709713.

17. Brasil. Decreto n. 968, de 7 de Maio de 1962. Dispõe sobre as normas técnicas para o tratamento da lepra. Diário Oficial da União 1962; 07 mai.

18. Brasil. Ministério da Saúde (MS). Fundação Nacional de Saúde. Gerência Nacional de Dermatologia Sanitária. Guia de Controle da Hanseníase. Brasília: MS; 1984.

19. Dantas JA. Incapacidades físicas em doentes de lepra no Estado de Sergipe. Bol Serv Nac Lepra 1969; 28(1):80-105.

20. Mello A, Fonte J. Estudo das incapacidades físicas (segundo classificação da Organização Mundial de Saúde) observadas por ocasião do fichamento, nos casos de lepra registrados em Santa Catarina - Brasil, de 1937 a 1966. Bol Serv Nac Lepra 1969; 28(1):40-64.

21. Belda W, Marlet P, Margarido LC, Sartori SG, Defonso $\mathrm{O}$. Inquérito sobre deformidades em hospital para doentes de hanseníase. Hansen Int 1977; 2(1):60-72.

22. Gonçalves A. Incapacidade em hanseníase: um estudo em nosso meio. Hansen Int 1979; 4(1):26-35.

23. Virmond MCL. Alguns apontamentos sobre a história da prevenção de incapacidades e reabilitação em hanseníase no Brasil. Hansen Int 2008; 33(2):13-18.

24. Brasil. Lei no 610, de 13 de janeiro de 1949. Fixa normas para profilaxia da lepra. Diário Oficial da União 1949; 02 fev.

25. Brasil. Portaria $n^{\circ} 165$, de 14 de maio de 1976. Dispõe sobre tratamento e profilaxia da hanseníase. Diário Oficial da União 1976; 14 maio.

26. Brasil. Ministério da saúde (MS). Secretária Nacional de Programas Especiais de Saúde. Divisão Nacional de Dermatologia Sanitária. Hanseníase: Prevenção e Tratamento das Incapacidades físicas, mediante técnicas simples. Rio de Janeiro: MS; 1977.

27. American Leprosy Missions (ALM). Instituto de Biomedicina de Caracas. Educación para la salud en la prevención de ladiscapacidad física en el enfermo de lepra. Caracas: ALM; 1993.

28. Brasil. Ministério da Saúde (MS). Fundação Nacional de Saúde. Coordenação Nacional de Dermatologia Sanitária. Manual de Prevenção de Incapacidades. Brasília: MS; 1997.

29. Brasil. Ministério da Saúde (MS). Secretaria de Vigilância em Saúde. Departamento de Vigilância Epidemiológica. Manual de prevenção de incapacidades. Brasília: MS; 2008.

30. Van Brakel WH, Saunderson VJ, Brandsma JW, Post E, Jellema R, Mcknight J. International workshop on neuropathology in leprosy -consensus report. Lepr Rev 2007; 78(4):416-433.

31. Brasil. Ministério da Saúde (MS). Manual de Prevenção de Incapacidades. Brasília: MS; 2001.

32. Brasil. Ministério da Saúde (MS). Guia de apoio para grupos de autocuidado em hanseníase. Brasília: MS; 2010.

33. Galan NGA, Beluci ML, Marciano LHSC, Prado RBR, Oliveira NGG, Bonini AG, Arakaki FR, Guimarães GS. Avaliação da prática do autocuidado em hanseníase. Hansen Int 2014; 39(2):27-35. 
34. Oliveira CR. Prevenção de Incapacidades na Hanseníase. In: Alves ED, Ferreira TL, Ferreira IN, organizadores. Hanseníase: avanços e desafios. Brasília: Nespron; 2014. p.259-295.

35. Brasil. Portaria Conjunta SVS/SAS no 125 , de 26 de março de 2009. Define as ações de controle da hanseníase. Diário Oficial da União 2009; 26 mar.

36. Brasil. Portaria GM/MS no 3.125, de 07 de outubro de 2010. Aprova as Diretrizes para Vigilância, Atenção e Controle da Hanseníase. Diário Oficial da União 2010; 7 out.

37. Brasil. Portaria GM/MS no 594, de 29 de outubro de 2010. Dispõe sobre a atenção integral em hanseníase. Diário Oficial da União 2010; 29 out.

38. Oliveira MLW, Grossi MA, Oliveira CF, Sena AS, Daxbacher E, Penna GO. Commitment to reducing disability: the Brazilian experience. Lepr Rev 2010; 81(4):324-345.

39. Gonçalves SD, Sampaio RF, Antunes CMF. Fatores preditivos de incapacidade em pacientes com hanseníase. Rev Saude Publica 2009; 42(2):267-274.

Artigo apresentado em 17/07/2018

Aprovado em 13/12/2018

Versão final apresentada em 15/12/2018 